\title{
1 Higher Variability in Fungi Compared to Bacteria in the Foraging Honey Bee Gut
}

2

13 Running Title (54 characters \& spaces): Fungi and bacteria in the honeybee gut

14 \#Address correspondence to Leslie E. Decker, lesldeck@stanford.edu.

15 *Present address: Department of Biology, University of Pittsburgh, 4200 Fifth Ave, Pittsburgh, 16 PA, USA.

17 MBio (Observation)

18 Abstract Word Count: 248

19 Main Text Word Count (excluding references, figure or table legends): 1190/1200 
Abstract (248/250):

21 Microbial communities in the honey bee gut have emerged as a model system to understand the effects of host-associated microbes on animals and plants. The specific distribution patterns of bacterial associates among honey bee gut regions remains a key finding within the field. The mid- and hindgut of foraging bees house a deterministic set of core species that affect host health. In contrast, the crop, or honey stomach, contains a more diverse set of bacteria that is highly variable in composition among individual bees. Whether this contrast between the two gut regions also applies to fungi, another major group of gut-associated microbes, remains unclear despite their potential influence on host health. In honey bees caught foraging at four sites across the San Francisco Peninsula, we found that fungi were much less distinct in species composition between the crop and the mid- and hindgut than bacteria. Unlike bacteria, fungi were highly variable in composition throughout the gut, and much of this variation was attributable to bee collection site. These patterns suggest that the fungi may be passengers rather than functionally significant gut symbionts. However, many of the fungi we found in the bees have been recognized as plant pathogens. Assuming that some fungi remain viable after passage through the gut, the distribution patterns we report here point to the potential importance of honey bees as vectors of fungal pathogens and suggest a more prominent role of honey bees in plant pathogen transmission than generally thought.

\section{Importance (Nontechnical explanation of why the work was undertaken; 150/150):} microbial communities. However, we have only begun to describe these fungi, much less examine their effects on most animals and plants. The honey bee, Apis mellifera, has emerged as a model system for studying host-associated microbes. Honey bees contain wellcharacterized bacteria specialized to inhabit different regions of the gut. Fungi also exist in the honey bee gut, but their composition and function remain largely undescribed. Here we show that, unlike bacteria, fungi vary substantially in species composition throughout the honey bee gut, contingent on where the bees are sampled. This observation suggests that fungi may be transient passengers and therefore unimportant as gut symbionts. However, our findings also indicate that honey bees could be major vectors of infectious plant diseases as many of the 
Gut microbes associated with the honey bee, Apis mellifera, have emerged as a powerful experimental system with which to uncover basic principles governing the assembly of hostassociated microbial communities and their effects on host health (1). Gut microbes can influence host health by modifying carbohydrate metabolism, protein degradation, and resistance to parasites $(2,3)$. Because each region of the honey bee gut houses distinct microbial communities, different gut regions, including the crop, the midgut, and the hindgut, must be examined separately to understand the functional contribution and assembly rules of each community $(4,5)$.

For example, in the mid- and hindgut (hereafter intestine) of the honeybee, a deterministic set of functionally indispensable core microbes appear to exist across all healthy workers regardless of location $(4,6,7)$. In contrast, the crop shows high heterogeneity in microbial species composition even among healthy workers, probably reflecting the spatial and temporal variation of ingested environmental microbes $(8,9)$ with potential consequences for host resistance to pathogens $(10,11)$. Critically, this research has focused almost exclusively on bacteria. It remains unknown whether the contrast between crop and intestinal communities applies only to bacteria or is also observed in other understudied groups of microbes, such as fungi, which may affect host health in ways that are currently underappreciated $(12,13)$.

In this study, we examined both bacteria and fungi in foraging workers to test two hypotheses: (1) fungal species composition is as distinct between the crop and the intestine as is bacterial species composition, and (2) fungi, like bacteria, show more variable species composition in the crop than in the intestine. To this end, we collected $101 \mathrm{~A}$. mellifera foraging workers at four sites on the San Francisco Peninsula in California, USA (Fig.1d, Table S1). We dissected the entire gut, separating the crop from the intestine. We then extracted and sequenced the bacterial V4 region of the $16 \mathrm{~S}$ ribosomal gene (505-806) and the fungal ITS1f-ITS2 region (14) (see Supplementary Information). Sequences were clustered into OTUs using VSEARCH (15), and taxonomy assigned for bacterial and fungal OTUs using QIIME (16) and UNITE (17).

As expected, bacterial community composition was most strongly predicted by gut region (PERMANOVA, gut region: $\mathrm{R}^{2}=0.32, \mathrm{p}=0.001$, Fig. $1 \mathrm{~b}-\mathrm{c}$ ), and higher among-host variation was detected in the crop than in the intestine (Betadeviation(18): $F_{1,168}=14.5, p=0.0002$ ). Although fungi also showed high variability in the crop (Fig. 1, Betadeviation: $F_{3,161}=40.8, p<0.0001$, Fig.S1), fungi in the intestine were more diverse in species composition than bacteria (Shannon: $F_{4,330}=7.60, p=0.006$, Fig.S2). Moreover, fungi retained more of the among-site differences from the crop to the intestine than did bacteria (Fig.1). Sample site was the strongest predictor of fungal species composition not just in the crop, but also in the intestine (PERMANOVA, site: $R^{2}=0.14, p=0.001$, Fig.1e-f), with gut region explaining only a small proportion of fungal composition (PERMANOVA, gut region: site, $R^{2}=0.03, p=0.001$ ).

To further quantify differences between bacteria and fungi, we applied the CLAM test, a multinomial species classification method (19), which differentiated OTUs into four categories:

94 crop-associated, intestine-associated, generalist, or too rare to classify. We found that only $1 \%$ of bacterial OTUs were categorized as generalists, whereas $7 \%$ of fungal OTUs fell into this 
category (Fig.2a-d). Furthermore, only $2 \%$ of bacterial OTUs were classified as intestineassociated, whereas $11 \%$ of fungal OTUs were classified as intestine-associated.

In addition, to determine how tightly crop composition was correlated with intestinal composition, we used Mantel tests to compare dissimilarity matrices between the crop and the intestine for bacteria and fungi separately. Crop composition was positively correlated with intestinal composition in both bacteria (Mantel $r=0.34, p<0.0001$, Fig.2e) and fungi (Mantel $r=0.35, p=<0.0001$, Fig.2f). The behavior of the fungi appeared nonnormal (Fig.2f), and we used quantile regression to determine how the composition relationships detected by the Mantel tests differed as dissimilarity values increased in magnitude from the mean. Bacteria in the intestine were correlated with those in the crop similarly across all three quantiles $\left(10^{\text {th }}\right.$ slope $=$ $0.12,50^{\text {th }}$ slope $=0.24,90^{\text {th }}$ slope $=0.27$, Fig. $2 \mathrm{~g}$ ), whereas in fungi, the slope of the relationship depended on the quantile examined $\left(10^{\text {th }}\right.$ slope $=1.14,50^{\text {th }}$ slope $=0.79,90^{\text {th }}$ slope $=0.07$, Fig. $\left.2 \mathrm{~h}\right)$. Lastly, we calculated checkerboard scores describing species co-occurrence (20), which indicated significant segregation only in the crop in bacteria, but this pattern was not observed in fungi (Table S2).

Taken together, our results reject both of the hypotheses we set out to test, highlighting contrasting compositional patterns between bacteria and fungi in the honey bee gut. Specifically, we found that honey bees retained more of the across-site differences from the crop to the intestine in fungi than in bacteria. Furthermore, unlike the constrained set of bacterial species in the intestine (4), fungal species composition was highly variable not just in the crop, but also in the intestine.

The broad distribution of fungal taxa we found throughout the gut suggests that these microbes may simply be ingested from external sources and then excreted. Moreover, our Mantel test results indicate that various fungal taxa disappear in a seemingly stochastic fashion as they move from the crop to the intestine. These results contrast the deterministic filtering of bacteria from the crop to the intestine that has been documented previously $(4,6)$ and corroborated here. The deterministic filtering in bacteria is also indicated by the disappearance of a significant checkerboard pattern of segregation from the crop to the intestine in bacteria, a pattern that we observed only in bacteria and not in fungi (Table S2).

Many fungal OTUs that could be identified to the species level in our study were reported previously as plant pathogens rather than honey bee symbionts (Table S3). Assuming that some of these fungi remain viable as they pass through the gut (21), our study suggests a more prominent role of honey bees as vectors of plant fungal pathogens across landscapes than previously recognized. Transmission of phytopathogens accumulating on the surface of honey bees has already been implicated in the spread of bacterial and fungal pathogens $(22,23)$, but the extent to which fecal transmission of fungal pathogens contributes to plant epidemics remains unknown. Honey bee hives are often transported among multiple orchards and farms for pollination (24). In our study, the composition of gut fungal communities was specific to foraging sites. However, if honey bees act as vectors of plant-pathogenic fungi, those fungal 
pathogens that would otherwise be locally restricted might be transmitted more broadly when hives are transported.

In summary, we provide evidence that fungal species composition is not nearly as distinct between the crop and intestine as in bacteria, and that fungal species composition is highly variable across the entire gut, unlike bacteria. These findings suggest that most fungi found in the honeybee gut may be transient passengers rather than symbionts that affect the health of the host. However, these fungi may still be functionally important as bee-transported plant pathogens. Further studies focused on the relationship between bees and gut fungi may increase our understanding of plant disease transmission and pollination.

Data availability. Upon acceptance, the 16s rRNA and ITS gene amplicon data sets generated by this study will be deposited and available in the Sequence Read Archives of the National Center for Biotechnology Information (NCBI).

\section{Supplemental Material}

154

Supplemental material is available online only. TEXT, DOCX file, $51 \mathrm{~KB}$ FIG S1, PDF file, 146 KB FIG S2, PDF file, $143 \mathrm{~KB}$ FIG S3, PDF file, $139 \mathrm{~KB}$ FIG S4, PDF file, $95 \mathrm{~KB}$ TABLE S1, PDF file, $60 \mathrm{~KB}$ TABLE S2, PDF file, $53 \mathrm{~KB}$ TABLE S3, PDF file, $66 \mathrm{~KB}$ TABLE S4, PDF file, $55 \mathrm{~KB}$

\section{Acknowledgements}

168 We thank Megan Morris for advice on DNA extraction, sequencing, and data analysis. We also 169 thank Callie Chappell, Nick Hendershot, Jesse Miller, and Chih-Fu Yeh for comments. This work 170 was funded by NSF (DEB 1737758). CED was supported by the Stanford Summer Research

171 Program.

The authors declare no conflict of interest. 


\section{References}

1. Zheng H, Steele MI, Leonard SP, Motta EVS, Moran NA. 2018. Honey bees as models for gut microbiota research. Lab Anim (NY). Nature Publishing Group.

2. Raymann K, Moran NA. 2018. The role of the gut microbiome in health and disease of adult honey bee workers. Curr Opin Insect Sci 26:97-104.

3. Zheng H, Perreau J, Elijah Powell J, Han B, Zhang Z, Kwong WK, Tringe SG, Moran NA. 2019. Division of labor in honey bee gut microbiota for plant polysaccharide digestion. Proc Natl Acad Sci 116:25909-25916.

4. Kwong WK, Moran NA. 2016. Gut microbial communities of social bees. Nat Rev Microbiol 14:374-384.

5. Engel P, Martinson VG, Moran NA. 2012. Functional diversity within the simple gut microbiota of the honey bee. Proc Natl Acad Sci 109:11002-11007.

6. Moran NA, Hansen AK, Powell JE, Sabree ZL. 2012. Distinctive gut microbiota of honey bees assessed using deep sampling from individual worker bees. PLoS One 7:e36393.

7. Kešnerová L, Emery O, Troilo M, Liberti J, Erkosar B, Engel P. 2020. Gut microbiota structure differs between honeybees in winter and summer. ISME J 14:801-814.

8. Corby-Harris V, Maes P, Anderson KE. 2014. The Bacterial Communities Associated with Honey Bee (Apis mellifera) Foragers. PLoS One 9:e95056.

9. Anderson KE, Sheehan TH, Mott BM, Maes P, Snyder L, Schwan MR, Walton A, Jones BM, Corby-Harris V. 2013. Microbial Ecology of the Hive and Pollination Landscape: Bacterial Associates from Floral Nectar, the Alimentary Tract and Stored Food of Honey Bees (Apis mellifera). PLoS One 8:e83125.

10. Arredondo D, Castelli L, Porrini MP, Garrido PM, Eguaras MJ, Zunino P, Antúnez K. 2018. Lactobacillus kunkeei strains decreased the infection by honey bee pathogens Paenibacillus larvae and Nosema ceranae. Benef Microbes 9:279-290.

11. Daisley BA, Pitek AP, Chmiel JA, Al KF, Chernyshova AM, Faragalla KM, Burton JP, Thompson GJ, Reid G. 2020. Novel probiotic approach to counter Paenibacillus larvae infection in honey bees. ISME J 14:476-491.

12. Stefanini I. 2018. Yeast-insect associations: It takes guts. Yeast 35:315-330.

13. Yun JH, Jung MJ, Kim PS, Bae JW. 2018. Social status shapes the bacterial and fungal gut communities of the honey bee. Sci Rep 8:1-11.

14. White TJ, Bruns T, Lee S, Taylor J. 1990. Amplification and direct sequencing of fungal ribosomal RNA genes for phylogenetics. PCR Protoc 18:315-322.

15. Rognes T, Flouri T, Nichols B, Quince C, Mahé F. 2016. VSEARCH: A versatile open source tool for metagenomics. PeerJ 4:e2584.

16. Caporaso JG, Kuczynski J, Stombaugh J, Bittinger K, Bushman FD, Costello EK. 2010. QIIME allows analysis of high-throughput community sequencing data. Nat Methods 7:335-336.

17. Nilsson RH, Larsson KH, Taylor AFS, Bengtsson-Palme J, Jeppesen TS, Schigel D, Kennedy P, Picard K, Glöckner FO, Tedersoo L, Saar I, Kõljalg U, Abarenkov K. 2019. The UNITE database for molecular identification of fungi: Handling dark taxa and parallel taxonomic classifications. Nucleic Acids Res 47:D259-D264.

18. Vannette RL, Fukami T. 2017. Dispersal enhances beta diversity in nectar microbes. Ecol Lett 20:901-910. 
19. Chazdon RL, Chao A, Colwell RK, Lin SY, Norden N, Letcher SG, Clark DB, Finegan B, Arroyo JP. 2011. A novel statistical method for classifying habitat generalists and specialists. Ecology 92:1332-1343.

20. Gotelli NJ. 2000. Null model analysis of species co-occurrence patterns. Ecology 81:26062621.

21. Parish JB, Scott ES, Correll R, Hogendoorn K. 2019. Survival and probability of transmission of plant pathogenic fungi through the digestive tract of honey bee workers. Apidologie 50:871-880.

22. Alexandrova M, Cimini B, Bazzi C, Carpana E, Massi S, Sabatini AG. 2002. The role of honeybees in spreading Erwinia amylovora, p. 55-60. In IX International Workshop on Fire Blight 590.

23. Gasparoto MCG, Lourenço SA, Tanaka FAO, Spósito MB, Marchini LC, Silva Junior GJ, Amorim L. 2017. Honeybees can spread Colletotrichum acutatum and C. gloeosporioides among citrus plants. Plant Pathol 66:777-782.

24. Rucker RR, Thurman WN, Burgett M. 2012. Honey bee pollination markets and the internalization of reciprocal benefits. Am J Agric Econ 94:956-977. 


\section{Figure Legends}

Figure 1. (a) Honeybee gut anatomy color-coded by sampling scheme dividing the gut into the crop (turquoise) and the remaining posterior intestine regions (gold). Non-metric multidimensional scaling (NMDS) based on Bray-Curtis dissimilarity matrices calculated from rarefied OTU tables show variation in (b) bacterial and (c) fungal community structure, where shape indicates collection site. Each dot indicates a bee individual. A map of the collection sites within the San Francisco Bay Area: Jasper Ridge Biological Preserve ( $\mathrm{N}=25$ bees), Stanford University ( $\mathrm{N}=26$ bees), Palo Alto ( $\mathrm{N}=24$ bees), and San Francisco ( $\mathrm{N}=26$ bees). (d). The same NMDS as in (b) and (c), but color-coded instead by collection site, illustrates weak retention of site effects in (e) bacteria and stronger site effects in (f) fungi.

Figure 2. Fewer bacterial OTUs were categorized as intestine-associated specialists and generalists compared to fungi using a multinomial species classification method (clamtest) to sort OTUs into categories based on relative abundance in each domain: (a) bacteria and (b) fungi. Summarized classification results for (c) bacteria and (d) fungi. Relationships between (e) bacterial crop and intestine community structure (dark green), and (f) fungal crop and intestine community structure (light green). Lines are regressions against the $10^{\text {th }}, 50^{\text {th }}$, and $90^{\text {th }}$ quantiles respectively from bottom to top. Changes in slope reflect the extent to which the composition of intestine communities depend on crop communities. Slope coefficients with 95\% confidence intervals for each quantile are plotted for $(\mathrm{g})$ bacteria and $(\mathrm{h})$ fungi in relation to the ordinary least square regression coefficient plotted with $95 \%$ confidence intervals in red. 
(a)

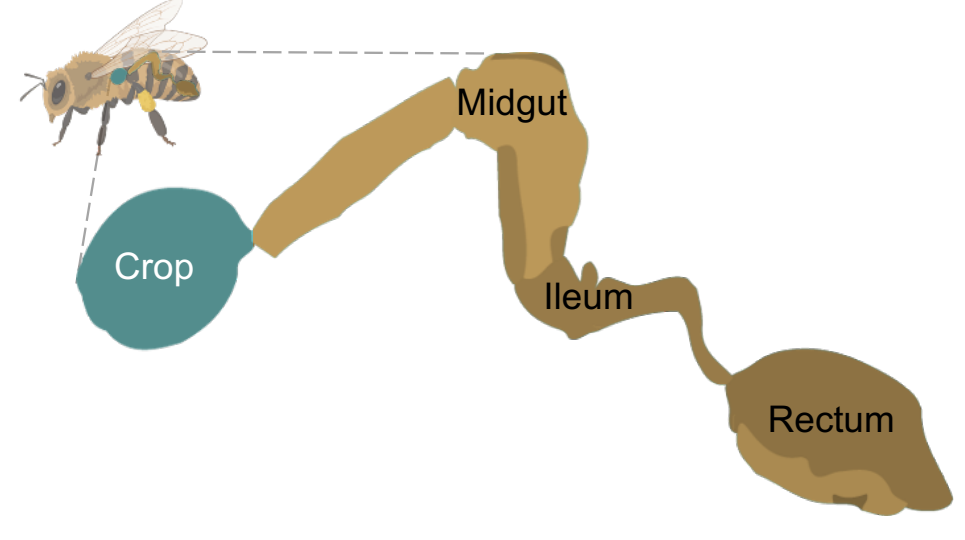

\section{Bacteria}

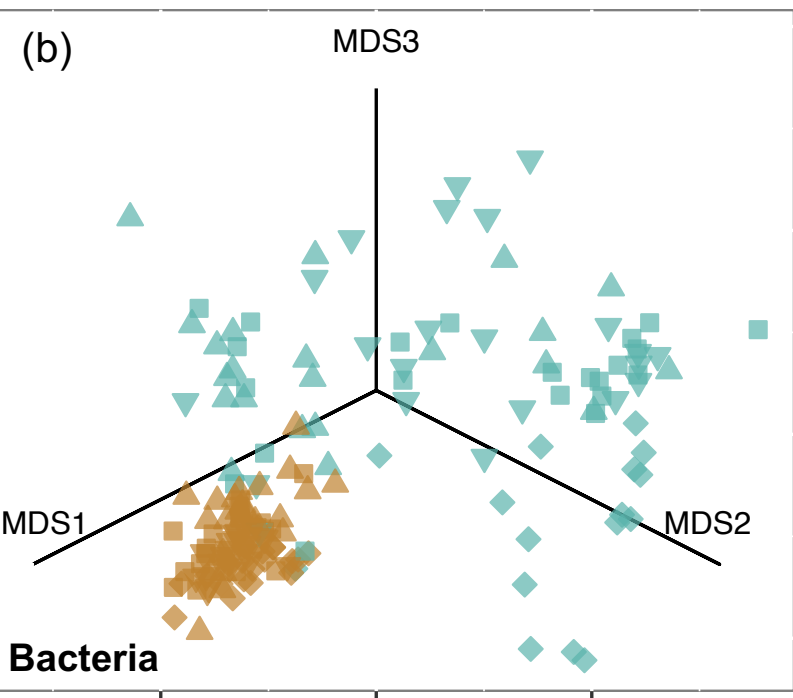

- Palo Alto $\nabla$ San Francisco (d) $37.8^{\circ} \mathrm{N}-$

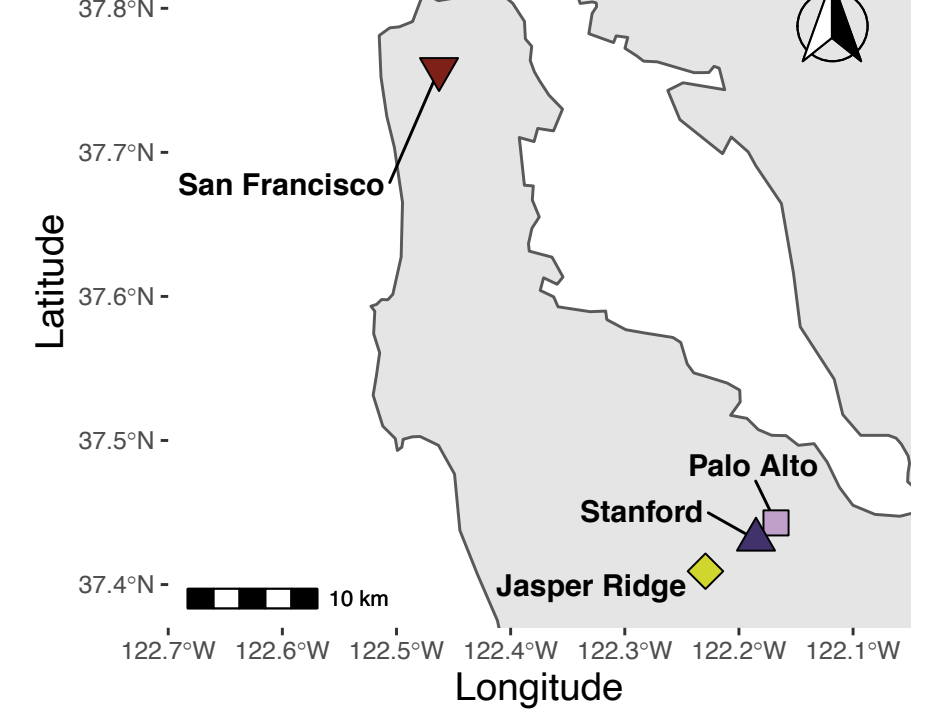

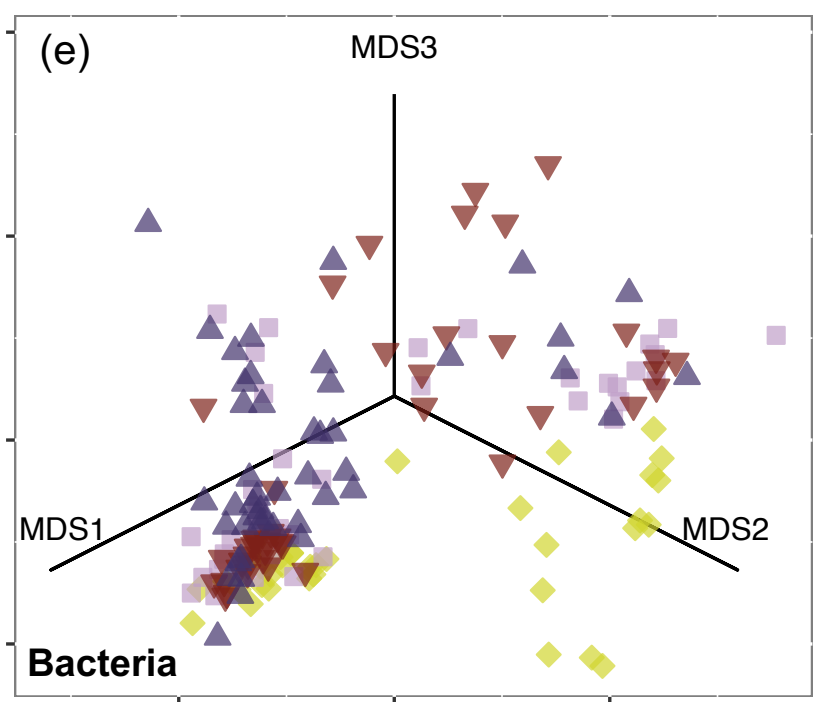

(c)

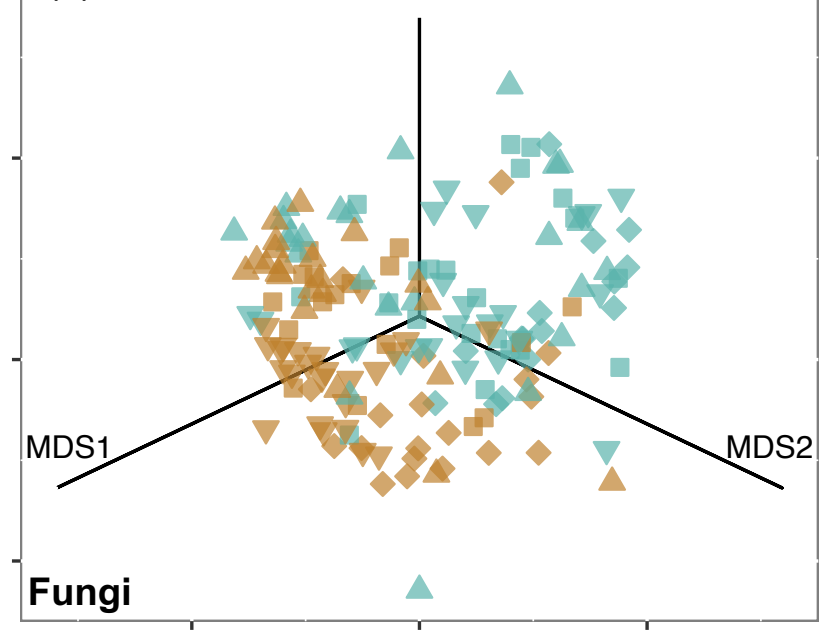

Fungi

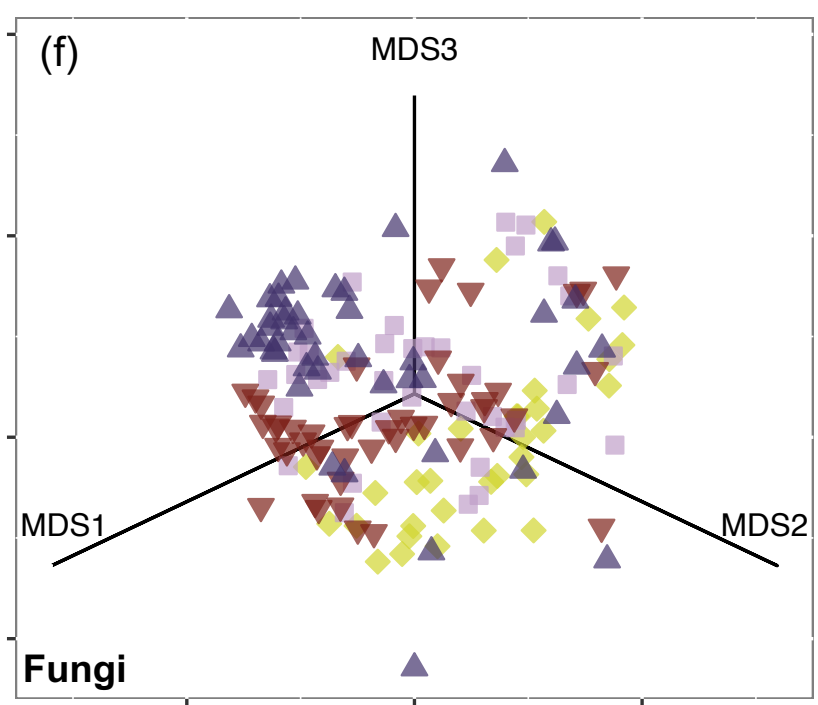


Generalist Specialist_Crop $\odot$ Specialist_Intestine Too_rare
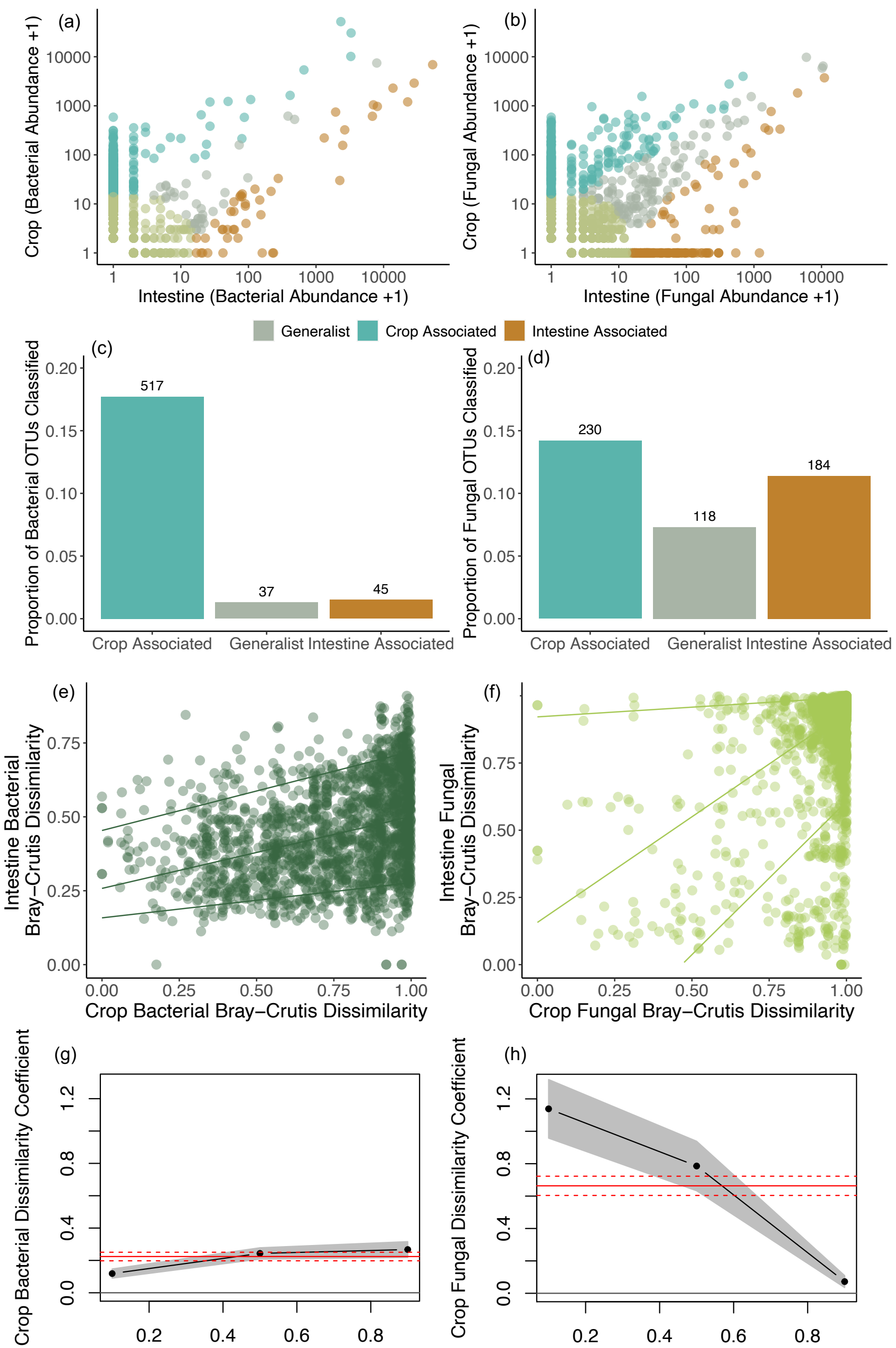

Percentile

Percentile 